\title{
Psychosocial management of chronic pain in patients with rheumatoid arthritis: challenges and solutions
}

\section{Louise Sharpe}

School of Psychology, The University of Sydney, Sydney, NSW, Australia
Correspondence: Louise Sharpe School of Psychology, Brennan MacCallum Building AI7, The University of Sydney, Sydney, NSW 2006, Australia Tel +6I 2 935। 4558

Email louise.sharpe@sydney.edu.au

\author{
This article was published in the following Dove Press journal: \\ Journal of Pain Research \\ I4 March 2016 \\ Number of times this article has been viewed
}

\begin{abstract}
There are numerous reviews and meta-analyses that confirm that psychological therapy is efficacious for patients with rheumatoid arthritis (RA) in terms of managing pain. Therefore, the literature has moved on to answer additional questions: 1) What types of interventions are most strongly supported by the current evidence? 2) Do different patients benefit from different approaches? 3) When is it best to intervene? 4) What modalities are best for administering the intervention? 5) What model of care should we be proposing that will result in widespread implementation and will ensure access for patients with RA? This review concludes that cognitive behavioral therapy (CBT) is the most efficacious treatment for pain management in RA; however, there are indications that mindfulness may have particular benefits for patients with a history of depression. CBT is most effective when administered early in the course of the disease. However, there is at present little evidence to confirm whether or not psychosocial interventions are effective for patients with comorbid psychological disorders. One of the major challenges is ensuring access to effective interventions for patients, particularly early on in the course of the disease, with a view to preventing physical and psychological morbidity. A stepped-care model is proposed; however, we urgently need more, better-quality trials of minimal interventions, particularly in Internet-delivered CBT, which appears promising and may form the cornerstone of future stepped-care models for providing psychosocial care to patients with RA.
\end{abstract}

Keywords: pain, pain management, cognitive behavioral therapy, psychosocial treatment, coping, psychosocial, psychotherapy

\section{Introduction}

Pain management programs, typically based on self-regulation or cognitive behavioral approaches, have a long history in the management of chronic pain. A description of the most commonly used interventions is listed in Table 1 . In the most recent Cochrane review, ${ }^{1}$ there was a clear efficacy of cognitive behavioral approaches to pain management on pain, disability, and mood. Although studies demonstrate the overall efficacy of these approaches, the effect sizes are small both at posttreatment and at follow-up. ${ }^{1-3}$ The fact that cognitive behavioral therapy (CBT) is efficacious for chronic pain is not a controversial claim, and researchers have now turned their attention to considering how the efficacy of existing programs can be improved. ${ }^{4}$ However, many of these studies include patients with heterogeneous chronic pain conditions, and only a small number of the trials have focused specifically on rheumatoid arthritis (RA).

There have been two specific meta-analyses of psychological interventions for RA and both of these confirmed evidence of efficacy of psychosocial interventions targeting 
Table I A list of interventions that have been trialed as psychosocial approaches to pain management for patients with RA, discussed in this review, a brief description, and the level of evidence

\begin{tabular}{|c|c|c|}
\hline Therapy & Description & Level of evidence and comments \\
\hline CBT & $\begin{array}{l}\text { CBT aims to identify unhelpful patterns of behavior and attitudes } \\
\text { toward RA and to change these. Hence, the behavioral components } \\
\text { include strategies to help achieve a balance of rest versus activity, } \\
\text { while the cognitive strategies aim to encourage an attitude of realistic } \\
\text { optimism toward the illness. CBT includes some or all of the following } \\
\text { strategies, including psychoeducation, relaxation, pacing and goal } \\
\text { setting, attention diversion, problem solving, assertiveness training, } \\
\text { cognitive challenging, and managing high-risk time and relapse. }\end{array}$ & $\begin{array}{l}\text { Level I evidence. } \\
\text { Definitely efficacious. } \\
\text { Demonstrated both in patients with chronic } \\
\text { pain generally and for patients with RA } \\
\text { specifically. } \\
\text { Dosage of at least six sessions is necessary. }\end{array}$ \\
\hline Expressive writing & $\begin{array}{l}\text { Participants are asked to write about stressful times in their lives as } \\
\text { a form of emotional expression. }\end{array}$ & $\begin{array}{l}\text { Level I evidence. } \\
\text { Definitely efficacious. } \\
\text { One head-to-head trial with CBT found CBT } \\
\text { was more effective than expressive writing. }\end{array}$ \\
\hline Mindfulness & $\begin{array}{l}\text { Mindfulness-based intervention teaches patients to adopt a } \\
\text { nonjudgmental and observant stance in relation to their experiences. } \\
\text { Although there are different variants of mindfulness, they typically } \\
\text { include a meditative component. Unlike CBT, mindfulness explicitly } \\
\text { encourages participants not to attempt to change, but rather to } \\
\text { accept their experiences. }\end{array}$ & $\begin{array}{l}\text { Level II evidence (Level I evidence for } \\
\text { chronic pain). } \\
\text { Definitely efficacious. } \\
\text { One head-to-head trial of CBT compared it } \\
\text { to mindfulness, where CBT outperformed } \\
\text { mindfulness in the whole sample. However, } \\
\text { patients with a history of clinical depression } \\
\text { did better with mindfulness. }\end{array}$ \\
\hline Problem solving & $\begin{array}{l}\text { Problem solving is often a component of CBT in the context of pain } \\
\text { management, but it can be a stand-alone treatment. Patients are taught } \\
\text { to identify problems, brainstorm potential solutions, evaluate each } \\
\text { solution, and then implement the solution and review its effect. }\end{array}$ & $\begin{array}{l}\text { No evidence for RA, specifically, but Level II } \\
\text { evidence for older patients who have arthritis } \\
\text { and a clinical depression. } \\
\text { Possibly efficacious. }\end{array}$ \\
\hline IFS-based psychotherapy & $\begin{array}{l}\text { IFS focuses on having patients attend to their experiences in a mindful } \\
\text { way and uses self-compassion to encourage dialogue with part of the }\end{array}$ & $\begin{array}{l}\text { Level II evidence. } \\
\text { Possibly efficacious. }\end{array}$ \\
\hline
\end{tabular}
self. Patients are taught to identify the thoughts and emotions that are associated with pain, fatigue, disability, and deformity and use an internal dialogue to respond to them.

ACT ACT shares overlap with both $C B T$ and mindfulness. ACT focuses on accepting internal experiences without judgment and on clarifying values that are important to an individual in order to commit to acting in accordance with one's values.

No evidence specifically for RA. Level II evidence for chronic pain. Definitely efficacious for chronic pain, unclear for RA.

One head-to-head trial of CBT and ACT for chronic pain, no differences between the treatments.

Notes: The following descriptors are used: Level I evidence: a systematic review or meta-analysis of RCTs is available; Level II: an RCT is available. Definitely efficacious: two or more RCTs from different researchers are available. Possibly efficacious: a single RCT or multiple RCTs from a single group of researchers are available.

Abbreviations: ACT, acceptance-and-commitment therapy; CBT, cognitive behavioral therapy; IFS, internal family systems; RA, rheumatoid arthritis; RCT, randomized controlled trial.

pain specifically in samples with RA. Astin et $\mathrm{al}^{2}$ found that psychological interventions led to improvements not only in pain and disability but also in psychological functioning, but with small effect sizes (Cohen's $d=0.18-0.27$ ). There was some indication that these programs could be more effective for patients earlier in the disease trajectory, but this could not be confirmed statistically. A more recent meta-analysis ${ }^{3}$ found similar results. That is, psychological interventions targeting self-regulation led to moderate effects on physical activity levels (Hedge's $g=0.45$ ) but smaller effects on pain, disability, anxiety and depression, and disability (Hedge's $g$ values $=0.17-0.38$ ), which were largely maintained at follow-up. Knittle et $\mathrm{al}^{3}$ also investigated moderators of outcome and found that interventions that included more components and treatments administered earlier in the course of RA were more efficacious.

Randomized controlled trials (RCTs) and meta-analyses confirming the efficacy of psychological interventions have led to guidelines that include the need for patients with RA to have access to psychological approaches as part of their disease management plans. For example, the recent European League Against Rheumatism (EULAR) ${ }^{5}$ recommendations indicate that patient education should be part of integrated care for all patients with RA based on Level I evidence. Similarly, British guidelines recommend access to multidisciplinary care for those in need in the first 2 years 
of RA. ${ }^{6}$ However, while these meta-analyses and reviews are able to determine whether, on average, psychosocial interventions are efficacious in the treatment of RA, the data leave a number of unanswered questions, which this review discusses.

First, there are insufficient data to determine on a meta-analytic basis whether or not one particular type of intervention is better than any other. Second, even if one intervention is more efficacious, on average, than another overall, do particular subgroups of patients preferentially respond to different approaches? Third, because the majority of studies focus on patients with RA regardless of the level of psychosocial distress, can we be sure that patients who present with comorbid psychological problems also benefit equally from these approaches? Fourth, although efficacious interventions exist, how can we meet the challenge of providing these interventions in a timely manner to patients with RA?

\section{Relative efficacy of different approaches}

Numerous approaches to pain management have been trialed, and a recent systematic review ${ }^{7}$ has attempted to determine which of these approaches is best supported by the literature. This review of 31 studies found consistent evidence for expressive writing and CBT of at least 6 weeks' duration, but it found mixed evidence for counseling, psychotherapy, mindfulness, and shorter-duration $\mathrm{CBT}^{7}$ These results are based on review and comparison of individual studies, and relatively few studies have directly compared more than one psychosocial treatment modality. However, those that have were able to determine differential efficacy of various treatment options.

Lumley et $\mathrm{al}^{8}$ compared the two interventions that were supported by the previously described systematic review.? In a $2 \times 2$ design, they randomized 264 adults with RA to receive a CBT oriented coping skills training program compared to arthritis education; and either a written emotional disclosure program or a controlled writing condition. This allowed the authors to determine whether each intervention was efficacious; and whether the combination was superior to either when administered alone. The results indicated that CBT resulted in decreased pain and improved psychosocial outcomes, which were maintained at 12-month follow-up. There were no interactions between written emotional disclosure and CBT, indicating that the inclusion of written emotional disclosure did not facilitate the efficacy of CBT. There were some effects of written emotional disclosure in the short-term, specifically on disease activity and disability, but pain also increased in those who received the written emotional disclosure intervention. Further, the positive results of written emotional disclosure were not maintained at follow-up. Hence, the authors concluded that CBT should be recommended to patients with RA.

While the results of this trial concur with those of a systematic review ${ }^{7}$ and the broader Cochrane review ${ }^{1}$ of psychological interventions for pain management, CBT is typically a broad-based approach that has many components. Interventions that fall under the umbrella of CBT, typically, have two important aims. These are to change behavior and to change people's beliefs or attitudes. In the case of RA, behavior change is usually helping patients to achieve a balance between rest and exercise, through strategies such as pacing, goal setting, problem solving, and relaxation strategies. Cognitive change helps patients to develop a more optimistic but realistic attitude toward the illness and to manage other stresses in their lives. For example, a typical CBT-oriented program for RA includes psychoeducation about RA, relaxation and attention diversion, goal setting and pacing, cognitive therapy including challenging thoughts, problem solving, and communication training, with skills in managing relapse. ${ }^{9}$ Some studies include all these components and others select a combination from among these. Therefore, although CBT appears to be the most efficacious approach to pain management in RA, it is unclear which strategies are most efficacious.

Sharpe and Schrieber ${ }^{10}$ conducted a dismantling trial in 104 patients with RA. They compared an intervention including the cognitive components of CBT with an intervention including the behavioral components with a broad-based CBT program. Contrary to expectations, they found that it was the group that received cognitive components of treatment that outperformed one of the other two active treatments on three of the seven outcomes. Specifically, those who received cognitive therapy or CBT outperformed those receiving only behavioral therapy and a wait list on tender joint counts and inflammation, as assessed by C-reactive protein, while cognitive therapy and behavioral therapy outperformed CBT and the wait list on anxiety. At 6-month follow-up, the only difference between groups was on tender joints again favoring the cognitive therapies. These results suggest that the cognitive components of intervention are important and that therefore researchers and clinicians need to ensure that a sufficient dosage of the cognitive components is included in broad-based CBT programs. Although CBT is the most strongly evidence-based intervention, to 
the author's knowledge, there are only two RCTs that have compared treatment efficacy for CBT with another credible psychological therapy for patients with RA.

Zautra et $\mathrm{al}^{11}$ compared a CBT program focused on pain reduction with a mindfulness-based stress-reduction intervention and an education-alone condition. They found that both the CBT program and the mindfulness program were more efficacious than education alone; however, there was evidence for the superiority of CBT on pain outcomes and inflammation for the whole sample $(n=144)$. Notably there were subgroup differences, which are discussed in the following paragraphs. However, in a more recent trial comparing the same three conditions, the same team ${ }^{12}$ reported that mindfulness and CBT were differentially effective on different outcomes. In 143 participants who completed daily diaries, they found that mindfulness produced the broadest results in terms of producing greater reductions in daily recorded pain, fatigue, and catastrophizing, as well as stress reactivity, perceived control, and disability in the morning. These large, well-controlled studies ${ }^{11,12}$ allow more confidence that mindfulness-based interventions are likely to be an efficacious alternative treatment for patients with RA and concur with the results of a recent systematic review and meta-analysis for pain management associated with conditions other than RA. ${ }^{13}$ Considering that there are relatively few studies of mindfulness in RA, available meta-analyses and systematic reviews have not been able to compare CBT with mindfulness. ${ }^{3,7}$ However, mindfulness-based interventions certainly show promise and may even be a preferred intervention under some circumstances.

\section{Are some interventions more effective for particular patients?}

The previously reviewed studies indicate that there is strong evidence for the efficacy of CBT as an effective intervention for managing pain in RA, and emerging evidence supports the use of mindfulness-based interventions. However, there are limitations to the literature. It is notable that the majority of studies that have been reviewed do not select patients who screen positive for high levels of distress or depression, yet depression affects a substantial minority of patients with RA. In fact, a meta-analysis of the rates of depression in RA indicates that $16.8 \%$ of patients meet the criteria for a major depressive episode, ${ }^{14}$ and many more have scores in the clinical range on questionnaires. This raises the possibility of whether or not the results of trials conducted in unselected patients are likely to be generalizable to patients who have comorbid psychological difficulties.
Zautra et $\mathrm{al}^{11}$ conducted subgroup analyses based on those patients who had a history of a major depressive episode compared to those patients with RA who did not. Although the main study results favored CBT for the entire sample, for those with a history of depression, mindfulness was more efficacious for those with a depression history on measures of affect and joint function. This result is consistent with well-replicated findings in the depression literature for the efficacy of mindfulness-based interventions in reducing relapse for depression among those with a history of depressive episodes. In a meta-analysis, it was found that mindfulness-based interventions reduced the risk of relapse in patients with a prior history of three or more episodes of depression by $43 \%$, but not in those with only two prior episodes. ${ }^{15}$ In the RA study, ${ }^{11}$ it was the presence of a current or previous diagnosis of depression (rather than a recurrent history) that led to better outcomes with mindfulness relative to CBT. It may be that the presence of a chronic physical illness in itself is a risk factor for the development of depression, and therefore, patients with a history of fewer episodes of depression are as likely to benefit from mindfulness as those who, in the absence of an illness, have a history of recurrent depression. While this is speculative, and requires replication, these results do suggest that if a patient with RA has a history of depressive episodes, mindfulness-based interventions should be considered, particularly if the patient has already had CBT and failed to benefit. However, although this study is helpful in elucidating the conditions under which CBT and mindfulness may be indicated, because participants were not currently depressed, whether either CBT or mindfulness alone is efficacious in patients currently depressed cannot be answered by this study.

\section{Psychosocial treatments for patients with comorbid psychological problems}

While there is evidence that psychosocial interventions improve depressive symptoms for people with RA, ${ }^{2,3}$ studies to date have not selected patients with comorbid depressive illness or elevated levels of distress. Yet, patients with RA commonly do have heightened levels of depressive illness, with a recent meta-analysis estimating that $16.8 \%$ of patients with RA meet criteria for a major depressive episode. ${ }^{14}$ Although anxiety disorders are less well investigated, available research suggests that heightened anxiety is even more common than elevated depression, ${ }^{16}$ and that comorbidity between the anxiety and depression is also common. ${ }^{17}$ Moreover, there is evidence that patients with high baseline levels 
of depression and anxiety do more poorly with psychosocial interventions, ${ }^{18}$ indicating that the approaches may be less effective with patients with high levels of depression or anxiety; or that these patients may need a larger dose of therapy than typically delivered in these programs. As such, it is an enormous limitation of the literature that, although the majority of studies do not exclude patients with anxiety and depressive disorders, patients are not selected on this basis. It is therefore possible that the approaches known to be effective for patients with RA are not effective in ameliorating the psychological disorders that are common in these groups.

Searches revealed a single study that had addressed the treatment of depression in RA specifically. Parker et a ${ }^{19}$ randomly allocated 54 patients with a major depressive disorder and RA to receive 1) CBT plus an antidepressant; 2) CBT plus a placebo; and 3) an attention placebo plus an antidepressant. In the main analyses, there were no differences among the groups, except that by 15-month follow-up, the patients who had received an antidepressant were more anxious than those who had not. In post hoc analyses, those who received an antidepressant did better than the patients in a group that declined antidepressant medication, who were used as a control group; however, the addition of CBT did not further enhance the effects of antidepressant medication. Unfortunately, the results from this study are difficult to interpret, considering the small sample size, nonrandomized control group, and null effect in the main preplanned analyses. However, these results do question whether interventions that were shown to be efficacious in people without depression would be equally effective in people with RA who are comorbidly depressed. However, searches of depression in the context of arthritis, generally (rather than RA specifically), give more reason for optimism.

As part of a large multicentered study (IMPACT), ${ }^{20}$ Lin et $\mathrm{a}^{21}$ investigated the efficacy of improving depression care for older adults with arthritis and a comorbid major depressive illness. The IMPACT study ${ }^{20}$ was a large study of 1,801 older adults ( $>60$ years of age) in 18 primary care practices who were randomized to receive screening and intervention for depression (where indicated) or usual care. Both medication (antidepressant medication) and/or psychological intervention (problem-solving therapy) were offered to affected patients in the active arm. ${ }^{20}$ In the 1,001 participants from the IMPACT study ${ }^{20}$ who had arthritis (the majority had osteoarthritis), improving the primary care management of depression through the use of antidepressant medication and/or problem-solving therapy led to reductions in depressive symptoms in the intervention group compared to the control group. ${ }^{21}$ In addition, there were gains in the intervention group for both pain and functional outcomes, including quality of life. Although encouraging, the majority of individuals in this study had osteoarthritis, rather than RA, and all were aged $>60$ years. Problem-solving therapy is known to be particularly efficacious in the management of older adults, which may be due to its particular suitability for individuals with early cognitive decline $\mathrm{e}^{22,23}$ and, as such, the generalization of these results to younger patients with RA is unknown. Further, $<50 \%$ of the intervention group received problem-solving therapy in the course of the trial; while in the larger sample, there was evidence that those who receive problem-solving therapy had improved depression outcomes,${ }^{20}$ whether this is true of the subsample with arthritis was unclear. Hence, there is a clear need for studies that demonstrate whether or not existing treatments that are most strongly evidence based (eg, CBT) for patients with RA are also effective for those with comorbid psychological difficulties or whether alternate approaches (such as problemsolving therapy or mindfulness) are more efficacious. In the meantime, it is therefore important that patients are offered psychological interventions relatively early in the course of their illness, ${ }^{6}$ which could help to prevent the development of depression.

\section{The benefits of early intervention}

The most recent meta-analysis of psychological interventions for patients with RA confirms that early intervention is more effective than intervening later in the course of RA. ${ }^{3}$ In RA, the first 2 years of illness is seen as particularly important for intervention, considering that RA is typically most active in terms of inflammation during this early period and therefore early intervention can prevent long-term damage. ${ }^{6}$ However, there is only one trial that has investigated the efficacy of psychological intervention (CBT) in this critical first 2-year period of the disease. Sharpe et $\mathrm{al}^{24}$ randomized 53 people with recently diagnosed RA to receive either CBT in addition to routine care or routine care alone. The short-term results showed that $\mathrm{CBT}$ was associated with improved joint functioning and depression in comparison to routine care. This cohort was followed up over 18 months, and there was evidence 18 months later of relative improvements in the CBT group compared to the control group in terms of disability and mood (both anxiety and depression), indicating that CBT had prevented a deterioration in disability and the development of clinically significant depressive symptoms. ${ }^{25}$ Further, 5 years later, the CBT group had used fewer health care resources, to the point that providing the intervention 
was more than offset by the savings to the health service. ${ }^{26}$ The difficulty with accessing incident cases of RA has precluded replication of these results, although other studies that have tried to intervene relatively early have also had promising results.

The only other trial that has specifically focused on early intervention (first 8 years) also investigated the efficacy of an individually tailored CBT program as an adjunct to routine care. ${ }^{27}$ In this study, participants chose two of four modules: pain, fatigue, negative mood, and social relationships. Interestingly, despite the typical focus on pain management in CBT programs, the most commonly chosen module was the fatigue module. Compared to routine care alone, the CBT group had reduced fatigue and depression and improved social support at posttreatment assessment. These results were also maintained at 6 months' follow-up.

These results are interesting because, although there has been an increase in recent years in research targeting fatigue as a primary outcome, a Cochrane review ${ }^{28}$ found that the evidence for psychosocial interventions effectively targeting fatigue remains low. However, other individual RCTs have shown a positive benefit of cognitive behavioral interventions targeting fatigue in patients with RA (eg, Hewlett et $\mathrm{al}^{29}$ ). Indeed, Hewlett et $\mathrm{al}^{29}$ found benefits not only on fatigue but also on pain associated with RA. Considering that fatigue is a common symptom of RA, the individualized approach that Evers et $\mathrm{al}^{27}$ developed may be a useful way forward for clinicians to target the symptoms of most concern for individual patients with RA. Unfortunately, although the results of the trial were positive, because the authors did not compare the individualized version of CBT with a standard protocol, it is difficult to know whether or not the individualization of the protocol added to its efficacy. Nonetheless, this trial certainly suggests that interventions targeting symptoms other than pain should be developed and evaluated, and that these could be used in conjunction with known effective interventions focusing on pain.

\section{How can we improve access to psychosocial care?}

Despite the positive results, reviewed earlier from individual RCTs, meta-analyses, systematic reviews, and consensus guidelines advocating for the availability of psychosocial interventions for patients with RA, in practice, most patients do not get access routinely to these programs, particularly not early in the course of their illness. In fact, there is evidence from large-scale surveys $(n=1,210)$ of patients with inflammatory arthritis that the majority would accept psychosocial programs with a focus on self-management if offered them (66\%); however, less than a quarter of these reported even being asked about psychosocial issues by a rheumatology professional (23\%). Most patients wanted help specifically with pain and fatigue ( $82 \%$ ) or managing the emotional consequences of living with RA (57\%) and its impact on work and leisure activities (52\%) ${ }^{30}$ However, these programs are not routinely available in most rheumatology settings.

The gap between the demand for psychological therapy and its availability is not limited to patients with RA. ${ }^{31}$ One model that has been proposed as a potential solution to this mismatch is a stepped-care approach. In a stepped-care model, brief but effective interventions are offered to a population universally (or to those who screened positive for distress), and only if patients fail to benefit from the brief approach requiring minimal resources are more intensive psychosocial services offered. The efficacy of a stepped-care model rests upon the efficacy of minimal interventions, which are typically those administered in the form of self-help or Internetdelivered interventions. Although in the RA literature, there is a long history of self-management approaches that have been shown to result in improvements in disability and pain, most of these are facilitated face-to-face in group formats. ${ }^{32}$ However, in a small, interview-based study, $78 \%$ of patients $(n=100)$ with either psoriatic arthritis or RA indicated that they would find an Internet-delivered psychosocial program to be acceptable. ${ }^{33}$ Given the increases in technology, if the interventions that have been found to be effective in faceto-face formats could be shown to be effective when administered over the Internet, this could make implementation of effective psychosocial interventions more likely. However, the RA literature in this regard is in its infancy.

In the more general pain management area, meta-analyses confirm the early potential of pain management approaches when delivered over the Internet, although the need for more and better-quality research trials has been stressed. ${ }^{34}$ Despite the need for additional and better-quality evidence, the meta-analysis revealed that Internet-delivered CBT (iCBT) programs were effective for patients with chronic pain in terms of pain severity and disability, although mood effects were less robust. ${ }^{34}$ A recent trial, however, which selected 52 patients with chronic pain on the basis of their heightened levels of distress, found significant impacts of iCBT on both mood and pain outcomes, indicating that interventions that specifically target psychological symptoms can be effective in the context of chronic pain. ${ }^{35}$ Importantly, from the point of view of a stepped-care model, a large recent trial of The Pain Course ${ }^{36}$ in 490 patients with chronic pain has shown that providing 
therapist support did not enhance the outcomes of an iCBT program for chronic pain. ${ }^{37}$ In that study, Dear et $\mathrm{al}^{36,37}$ found similar results in terms of completion of the Pain Course, regardless of whether therapist support was routinely offered, offered in response to a request from the participant, or not offered at all. Further, outcomes were also indistinguishable from the three conditions. This is important because reducing the requirement for a therapist to support patients with weekly telephone contact through a program is likely to considerably improve the likelihood of implementation of Internetbased programs. Hence, if these results could be shown to be generalizable to those with RA, iCBT could become an important solution for increasing the availability of effective, and universal, psychosocial care for patients.

There have now been a few trials of Internet-delivered therapy for RA. Shigaki et $\mathrm{al}^{38}$ found that patients with RA who volunteered for the Internet-based intervention were highly adherent and the majority completed intervention. Although improvements were seen in quality of life and selfefficacy at posttreatment evaluation, which were maintained at follow-up, there were no improvements seen in measures of disability or mood. Trudeau et $\mathrm{al}^{39}$ found similar results in a study of $\mathrm{iCBT}$ in patients with arthritis. That is, improvements in coping strategies and self-efficacy were observed, but outcomes such as disability and mood were not found to be improved following treatment. Although these results are encouraging, the sample from the study by Trudeau et $\mathrm{al}^{39}$ was a group of patients who predominantly had osteoarthritis, and therefore, it is not clear how generalizable these results are to patients with RA. However, Internet-delivered interventions specifically targeting an increase in physical activity have been found to be efficacious for patients with RA, resulting in significantly greater increases in the primary outcome of physical activity. ${ }^{40}$ Overall, these results are consistent with a systematic review ${ }^{41}$ of Internet-delivered self-help psychological interventions for people with health problems, which found that there was moderate evidence for efficacy in pain and overall promising results for Internet-delivered interventions across health conditions, with the exception of diabetes. Nonetheless, there is currently insufficient evidence to confirm that Internet-delivered approaches in RA are sufficiently well supported to advocate their use as a first line of treatment; however, further research into such approaches are urgently needed.

\section{Future directions}

Despite the efficacy of psychological treatments, particularly CBT, for pain management in patients with RA, the programs provide on average only small gains in the primary outcomes. ${ }^{1-3}$ This is likely because patients with RA have different needs. Some have difficult psychosocial issues even early in the illness, ${ }^{42,43}$ while others do not have elevated levels of distress. Therefore, one would not necessarily expect that large effects in psychological outcomes would be observed for the entire group. Further, some patients respond well to tight medical control early in the course of the illness and have relatively low levels of inflammation and even achieve remission, ${ }^{44}$ whereas others have high levels of pain and inflammation. Again, the combination of patients in these trials with good disease outcomes, as well as those who are very disabled by their illness, probably lowers the effect sizes that can realistically be achieved. Nonetheless, even taking these factors into account, on the basis of the very small effect sizes achieved (particularly in the long-term), most would argue that there is considerable room for improvement in our protocols. As indicated in this review, CBT is the most widely used and evidence-based intervention for pain management. ${ }^{1}$ It is possible that there are ways to increase the efficacy of existing programs, such as by increasing the dose of effective components (such as cognitive therapy, as described by Sharpe and Schrieber ${ }^{10}$ ) or by adding to existing protocols.

One area that has been largely neglected in the field of pain management is the inclusion of family members in pain management approaches. There is only one study that has investigated the efficacy of the inclusion of family members in behavioral intervention for patients with RA. ${ }^{45}$ This study showed that while both versions of the intervention were effective compared to control, the behavioral program that involved families was more efficacious in the short-term than the same program without family involvement. However, by 6-month follow-up, the two programs were equally efficacious. ${ }^{41}$ Nonetheless, considering that this was a small study, it seems probable that it was underpowered to detect differences between groups, and these results suggest that further research focusing on family-based interventions would be worthwhile. This is particularly the case because research suggests that those individuals who experience the highest level of depressive symptoms typically report low levels of satisfaction with their social support. ${ }^{45}$ However, there may also be other interventions that encourage individuals from a family systems approach without necessarily requiring family members to be present for therapy.

A recent proof-of-concept study investigated the efficacy of an internal family systems (IFS)-based psychotherapy in RA. ${ }^{46}$ IFS focuses on having patients attend to their experiences in a 
mindful way and uses self-compassion to encourage dialogue with part of the self. Patients are taught to identify the thoughts and emotions that are associated with pain, fatigue, disability, and deformity and use an internal dialogue to respond to them. In comparison to a group that completed an educational program, participants in the IFS group reported improvements in pain and physical function. Further, at 1-year follow-up, benefits remained in pain and depression. Interestingly, IFS shares a number of components with traditional CBT, and in particular, mindfulness and acceptance-and-commitment therapy (ACT, often referred to as the third wave in $\mathrm{CBT}^{47}$ ). For example, both IFS and CBT use strategies that result in identifying and subsequently distancing oneself from one's thoughts and feelings. For CBT, identifying patterns between thoughts and feelings and challenging beliefs lies at the core of CBT for RA. The main difference between CBT and IFS is that in CBT, there is a focus on challenging the content of thoughts to be more helpful and realistic, whereas in IFS, the compassionate voice is evoked, which may or may not specifically challenge the content of the thought. ${ }^{48}$ However, some recent variants of CBT, such as compassion-focused therapy, use similar strategies. ${ }^{49}$ There are also overlaps with ACT, which has been gaining in popularity in recent years as an approach to pain management. ${ }^{13}$ ACT focuses on accepting internal experiences without judgment and clarifying values that are important to an individual in order to commit to acting in accordance with one's values. There are many similarities between ACT and CBT, but one major difference is that ACT explicitly does not challenge cognitive content but, rather, relies on a process of diffusion, which encourages patients to accept their thoughts as observations, nonjudgementally.

Evidence to date supports the efficacy of ACT for pain management; however, Veehof et $\mathrm{al}^{13}$ were only able to identify three RCTs on the role of ACT in pain management in their systematic review and meta-analysis of third wave treatments. Further, none of these was in patients with RA. There has since been another large, well-conducted RCT that compared ACT with CBT. ${ }^{50}$ This study found that both treatments led to comparable improvements in patients with chronic pain, although there was some evidence to indicate that patients may have had a preference for ACT over CBT. While these data are encouraging, there are currently insufficient data to recommend ACT for the management of pain in patients with RA, because these patients were ones with heterogenous chronic pain conditions. It is important to point out that in the area of anxiety, for which CBT and ACT have shown equivalence at posttreatment and in early follow-ups, long-term follow-ups have demonstrated the advantage of cognitive approaches over the long-term, because the effects of CBT tend to increase over time and the opposite is true for ACT., ${ }^{7,51}$ Second, ACT is yet to be used specifically with patients with RA.

\section{Conclusion and recommendations}

In conclusion, there is now no doubt that psychological intervention for the management of pain in patients with RA is efficacious and therefore can be considered to be evidence based. ${ }^{1-3}$ Further, the most strongly evidence-based treatment is $\mathrm{CBT}^{7}$ and it is most efficacious if offered early in the course of the disease. ${ }^{3}$ Indeed, the evidence for psychosocial interventions as an adjunct to medical management in patients with RA has led various international bodies to recommend that patients be given access to these interventions, early in the course of their illness. ${ }^{5,6}$ However, implementation of routine psychosocial intervention services has been less successful and remains a challenge, and the majority of patients typically report not having even discussed the psychological challenges of living with RA with their treating physicians. ${ }^{30}$ Hence, the primary challenge is to determine how relatively expensive interventions (eg, face-to-face psychological treatments of at least six sessions) can be made more accessible to patients with RA from the time when they are diagnosed.

In other settings, such as cancer care, stepped-care programs usually involve first screening patients for high levels of distress. For those who screen positive, a clinical assessment is conducted to determine whether or not a psychological disorder or unmet psychological need is present. Where an unmet psychological need is identified, patients are then offered a less-resource-intensive intervention in the first instance. Those patients who fail to derive benefit from the less intensive intervention, are then offered the full face-to-face intensive intervention, as required (see protocols for ongoing clinical trials ${ }^{53,54}$ ). However, there are problems with simply adopting this model and translating it into practice in RA.

First, there is no evidence, as previously discussed, that psychological interventions are more efficacious for patients who are highly distressed. Therefore, screening for depression and distress may be unnecessary in patients with RA because evidence is not available to suggest that interventions are effective in patients with psychological difficulties, in addition to RA. Rather, the evidence would support the universal application of less-resource-intensive options (eg, Internet-based approaches) for patients as close to diagnosis as possible. These interventions would focus on helping patients to manage their illness, as well as to learn coping strategies for managing pain and other symptoms and for 
managing the emotional consequences of living with $\mathrm{RA}^{24-26}$ with a view to preventing the development of psychological difficulties associated with RA. Those patients, who over the course of the illness subsequently become distressed, could then be offered more-intensive versions of psychosocial interventions (eg, face-to-face sittings).

While this model is intuitively appealing, it is urgent that we get more data on the efficacy of less-resource-intensive interventions, such as Internet-based therapy, specifically for RA. Until such time as we have efficacious interventions that require few resources, such a stepped-care model would be premature. Indeed, the success of such a model would rely on a number of factors that are not yet known: 1) the efficacy of the low-resource intervention; 2) the degree to which participants were willing to engage in the intervention; and 3) the subsequent identification of those patients who require psychosocial intervention and support following the minimal intervention. However, implementation remains the leading problem in ensuring that patients with RA have their psychosocial needs met, and therefore, it is important to ensure that evidence-based psychosocial interventions are translated into optimal service provision for patients.

\section{Disclosure}

Professor Sharpe is paid on a casual basis to deliver workshops for local and international conferences concerning the role of cognitive behavioral therapy in facilitating adjustment to illness or in the treatment of chronic pain. The author reports no other conflicts of interest in this work.

\section{References}

1. Williams A, Eccleston C, Morley S. Psychological therapies for the management of chronic pain (excluding headache) in adults. Cochrane Database Syst Rev. 2012;11:CD007407.

2. Astin J, Beckner W, Soeken K, Hochberg MC, Berman B. Psychological interventions for rheumatoid arthritis: a meta analysis of randomized controlled trials. Arthritis Rheum. 2002;47(3):291-302.

3. Knittle K, Maes S, De Gucht V. Psychological interventions for rheumatoid arthritis: examining the role of self-regulation with a systematic review and meta-analysis of randomized controlled trials. Arthritis Care Res (Hoboken). 2010;62(10):1460-1472.

4. Ehde DM, TM Dillworth, JA Turner. Cognitive-behavioral therapy for individuals with chronic pain: efficacy, innovations, and directions for research. American Psychologist. 2014;69(2):153.

5. Zangi HA, Ndosi M, Adams J, et al; European League Against Rheumatism (EULAR). EULAR recommendations for patient education for people with inflammatory arthritis. Ann Rheum Dis. 2015;74(6):954-962.

6. Luqmani R, Hennell S, Estrach C, et al; British Society for Rheumatology; British Health Professionals in Rheumatology Standards, Guidelines and Audit Working Group. British society for rheumatology and british health professionals in rheumatology guideline for the management of rheumatoid arthritis (the first two years). Rheumatology. 2006;45(9):1167-1169.
7. Dissanayake RK, Bertouch JV. Psychosocial interventions as adjunct therapy for patients with rheumatoid arthritis: a systematic review. Int J Rheum Dis. 2010;13(4):324-334.

8. Lumley MA, Keefe FJ, Mosley-Williams A, et al. The effects of written emotional disclosure and coping skills training in rheumatoid arthritis: a randomized clinical trial. J Consult Clin Psychol. 2014;82(4):644.

9. Sharpe L, Sensky T, Timberlake N, Allard S, Brewin CR. The role of cognitive behavioural therapy in facilitating adaptation to illness in rheumatoid arthritis: a case series. Behav Cogn Psychother. 2001; 29(03):303-309.

10. Sharpe L, Schrieber L. A blind randomized controlled trial of cognitive versus behavioral versus cognitive-behavioral therapy for patients with rheumatoid arthritis. Psychother Psychosom. 2012;81(3): 145-152.

11. Zautra AJ, Davis MC, Reich JW, et al. Comparison of cognitive behavioral and mindfulness meditation interventions on adaptation to rheumatoid arthritis for patients with and without history of recurrent depression. J Consult Clin Psychol. 2008;76(3):408.

12. Davis MC, Zautra AJ, Wolf LD, Tennen H, Yeung EW. Mindfulness and cognitive-behavioral interventions for chronic pain: differential effects on daily pain reactivity and stress reactivity. J Consult Clin Psychol. 2015;83(1):24.

13. Veehof MM, Oskam MJ, Schreurs KM, Bohlmeijer ET. Acceptancebased interventions for the treatment of chronic pain: a systematic review and meta-analysis. Pain. 2011;152(3):533-542.

14. Matcham F, Rayner L, Steer S, Hotopf M. The prevalence of depression in rheumatoid arthritis: a systematic review and meta-analysis. Rheumatology. 2013;52(12):2136-2148.

15. Piet J, Hougaard E. The effect of mindfulness-based cognitive therapy for prevention of relapse in recurrent major depressive disorder: a systematic review and meta-analysis. Clin Psychol Rev. 2011;31(6): 1032-1040.

16. Murphy LB, Sacks JJ, Brady TJ, Hootman JM, Chapman DP. Anxiety and depression among US adults with arthritis: prevalence and correlates. Arthritis Care Res (Hoboken). 2012;64(7):968-976.

17. Covic T, Cumming SR, Pallant JF, et al. Depression and anxiety in patients with rheumatoid arthritis: prevalence rates based on a comparison of the Depression, Anxiety and Stress Scale (DASS) and the hospital, Anxiety and Depression Scale (HADS). BMC Psychiatry. 2012;12(1):6.

18. Matcham F, Norton S, Scott DL, Steer S, Hotopf M. Symptoms of depression and anxiety predict treatment response and long-term physical health outcomes in rheumatoid arthritis: secondary analysis of a randomized controlled trial. Rheumatology. 2015. Epub 2015 Sep 8.

19. Parker JC, Smarr KL, Slaughter JR, et al. Management of depression in rheumatoid arthritis: a combined pharmacologic and cognitivebehavioral approach. Arthritis Rheum. 2003;49(6):766-777.

20. Arean P, Hegel M, Vannoy S, Fan MY, Unuzter J. Effectiveness of problem-solving therapy for older, primary care patients with depression: results from the IMPACT project. Gerontologist. 2008;48(3):311-323.

21. Lin EH, Katon W, Von Korff M, et al. Effect of improving depression care on pain and functional outcomes among older adults with arthritis: a randomized controlled trial. JAMA. 2003;290(18):2428-2429.

22. Alexopoulos GS, Raue PJ, Kiosses DN, et al. Problem-solving therapy and supportive therapy in older adults with major depression and executive dysfunction: effect on disability. Arch Gen Psychiatry. 2011;68(1):33-41.

23. Areán PA, Raue P, Mackin RS, Kanellopoulos D, McCulloch C, Alexopoulos GS. Problem-solving therapy and supportive therapy in older adults with major depression and executive dysfunction. Am J Psychiatry. 2010;167(11):1391-1398.

24. Sharpe L, Sensky T, Timberlake N, Ryan B, Brewin CR, Allard S. A blind, randomized, controlled trial of cognitive-behavioural intervention for patients with recent onset rheumatoid arthritis: preventing psychological and physical morbidity. Pain. 2001;89(2-3): 275-283. 
25. Sharpe L, Sensky T, Timberlake N, Ryan B, Allard S. Long-term efficacy of a cognitive behavioural treatment from a randomized controlled trial for patients recently diagnosed with rheumatoid arthritis. Rheumatology. 2003;42(3):435-441.

26. Sharpe L, Allard S, Sensky T. Five-year followup of a cognitivebehavioral intervention for patients with recently-diagnosed rheumatoid arthritis: effects on health care utilization. Arthritis Rheum. 2008;59(3):311-316.

27. Evers AW, Kraaimaat FW, van Riel PL, de Jong AJ. Tailored cognitivebehavioral therapy in early rheumatoid arthritis for patients at risk: a randomized controlled trial. Pain. 2002;100(1):141-153.

28. Cramp F, Hewlett S, Almeida C, et al. Non-pharmacological interventions for fatigue in rheumatoid arthritis. Cochrane Database Syst Rev. 2013;8:CD008322.

29. Hewlett S, Ambler N, Almeida C, et al. Self-management of fatigue in rheumatoid arthritis: a randomised controlled trial of group cognitivebehavioural therapy. Ann Rheum Dis. 2011;70(6):1060-1067.

30. Dures E, Almeida C, Caesley J, et al. Patient preferences for psychological support in inflammatory arthritis: a multicentre survey. Ann Rheum Dis. 2014. Epub 2014 Sep 26.

31. Bower P, Gilbody S. Stepped care in psychological therapies: access, effectiveness and efficiency narrative literature review. Br J Psychiatry. 2005;186(1):11-17.

32. Warsi A, Wang PS, LaValley MP, Avorn J, Solomon DH. Selfmanagement education programs in chronic disease: a systematic review and methodological critique of the literature. Arch Intern Med. 2004;164(15):1641-1649.

33. Ferwerda M, van Beugen S, van Burik A, et al. What patients think about E-health: patients' perspective on internet-based cognitive behavioral treatment for patients with rheumatoid arthritis and psoriasis. Clin Rheumatol. 2013;32(6):869-873.

34. Bender JL, Radhakrishnan A, Diorio C, Englesakis M, Jadad AR. Can pain be managed through the internet? A systematic review of randomized controlled trials. PAIN. 2011;152(8):1740-1750.

35. Buhrman M, Syk M, Burvall O, Hartig T, Gordh T, Andersson G. Individualized guided internet-delivered cognitive-behavior therapy for chronic pain patients with comorbid depression and anxiety: a randomized controlled trial. Clin J Pain. 2015;31(6):504-516.

36. Dear BF, Titov N, Perry KN, et al. The pain course: a randomised controlled trial of a clinician-guided internet-delivered cognitive behaviour therapy program for managing chronic pain and emotional well-being. PAIN. 2013;154(6):942-950.

37. Dear BF, Gandy M, Karin E, et al. The pain course: a randomised controlled trial examining an internet-delivered pain management program when provided with different levels of clinician support. Pain. 2015;156(10):1920-1935

38. Shigaki CL, Smarr KL, Siva C, Ge B, Musser D, Johnson R. RAHelp: an online intervention for individuals with rheumatoid arthritis. Arthritis Care Res (Hoboken). 2013;65(10):1573-1581.

39. Trudeau KJ, Pujol LA, DasMahapatra P, Wall R, Black RA, Zacharoff K. A randomized controlled trial of an online self-management program for adults with arthritis pain. J Behav Med. 2015;38(3):483-496.

40. Van den Berg M, Ronday HK, Peeters AJ, et al. Using internet technology to deliver a home-based physical activity intervention for patients with rheumatoid arthritis: a randomized controlled trial. Arthritis Rheum. 2006;55(6):935-945.

Journal of Pain Research

\section{Publish your work in this journal}

The Journal of Pain Research is an international, peer-reviewed, open access, online journal that welcomes laboratory and clinical findings in the fields of pain research and the prevention and management of pain. Original research, reviews, symposium reports, hypothesis formation and commentaries are all considered for publication.
41. Beatty L, Lambert S. A systematic review of internet-based self-help therapeutic interventions to improve distress and disease-control among adults with chronic health conditions. Clin Psychol Rev. 2013;33(4):609-622.

42. Dobkin PL, Filipski M, Looper K, et al; McGill Early Arthritis Research Group. Identifying target areas of treatment for depressed early inflammatory arthritis patients. Psychother Psychosom. 2008;77(5):298-305.

43. Gåfvels C, Hägerström M, Nordmark B, Wändell PE. Psychosocial problems among newly diagnosed rheumatoid arthritis patients. Clin Rheumatol. 2012;31(3):521-529.

44. Schipper LG, Vermeer M, Kuper HH, Hoekstra MO, Haagsma CJ, Fransen J. A tight control treatment strategy aiming for remission in early rheumatoid arthritis is more effective than usual care treatment in daily clinical practice: a study of two cohorts in the Dutch Rheumatoid Arthritis Monitoring registry. Ann Rheum Dis. 2012;71(6):845-850.

45. Radojevic V, Nicassio PM, Weisman MH. Behavioral intervention with and without family support for rheumatoid arthritis. Behav Ther. 1993;23(1):13-30.

46. Shadick NA, Sowell NF, Frits ML, et al. A randomized controlled trial of an internal family systems-based psychotherapeutic intervention on outcomes in rheumatoid arthritis: a proof-of-concept study. J Rheumatol. 2013;40(11):1831-1841.

47. Kahl KG, Winter L, Schweiger U. The third wave of cognitive behavioural therapies: what is new and what is effective? Curr Opin Psychiatry. 2012;25(6):522-528.

48. Sharpe L. Psychological treatment for rheumatoid arthritis works: now we need to know what elements are most effective and for whom. J Rheumatol. 2013;40(11):1788-1790.

49. Gilbert P. Introducing compassion-focused therapy. Adv Psychiatr Treat. 2009;15(3):199-208.

50. Wetherell JL, Afari N, Rutledge T, et al. A randomized, controlled trial of acceptance and commitment therapy and cognitive-behavioral therapy for chronic pain. Pain. 2011;152(9):2098-2107.

51. Forman EM, Shaw JA, Goetter EM, Herbert JD, Park JA, Yuen EK. Long-term follow-up of a randomized controlled trial comparing acceptance and commitment therapy and standard cognitive behavior therapy for anxiety and depression. Behav Ther. 2012;43(4):801-811.

52. Krebber AM, Leemans CR, de Bree R, et al. Stepped care targeting psychological distress in head and neck and lung cancer patients: a randomized clinical trial. BMC Cancer. 2012;12(1):173.

53. Mattsson S, Alfonsson S, Carlsson M, Nygren P, Olsson E, Johansson B. U-CARE: internet-based stepped care with interactive support and cognitive behavioral therapy for reduction of anxiety and depressive symptoms in cancer-a clinical trial protocol. BMC Cancer. 2013; 13(1):414

54. Unützer J, Katon W, Williams JW Jr, et al. Improving primary care for depression in late life: the design of a multicenter randomized trial. Med Care. 2001;39(8):785-799.

The manuscript management system is completely online and includes a very quick and fair peer-review system, which is all easy to use. Visit http://www.dovepress.com/testimonials.php to read real quotes from published authors. 